\title{
ON FORMAL LOCAL COHOMOLOGY MODULES WITH RESPECT TO A PAIR OF IDEALS
}

\author{
T.H. FREITAS AND V.H. JORGE PÉREZ
}

\begin{abstract}
We introduce a generalization of the formal local cohomology module, which we call a formal local cohomology module with respect to a pair of ideals, and study its various properties. We analyze their structure, upper and lower vanishing and non-vanishing properties. There are various exact sequences concerning formal cohomology modules, among them we have a Mayer-Vietoris sequence with respect to pair ideals. Also, we give another proof for a generalized version of the local duality theorems for Gorenstein, CohenMacaulay rings, and a generalization of the Grothendieck duality theorem for Gorenstein rings. We discuss the concept of formal grade with respect to a pair of ideals and give some results about this.
\end{abstract}

1. Introduction. Throughout this paper, $R$ is a commutative Noetherian (non-zero identity) ring, and $\mathfrak{a}, \mathfrak{b}, I$ and $J$ are ideals of $R$. Let $M$ be a finitely generated $R$-module. It is well known that, for $i \in \mathbb{N}$, $H_{\mathfrak{a}}^{i}(M)$ denotes the $i$ th local cohomology module of $M$ with respect to $\mathfrak{a}$, see $[4,10,14]$.

When $(R, \mathfrak{m})$ is a local ring, Schenzel [19] defined an object of study as follows. Let

$$
\underline{x}=x_{1}, \ldots, x_{r}
$$

be a system of elements of $R$ with $\mathfrak{b}=\operatorname{Rad}(\underline{x} R)$, and let $\check{C}_{\underline{x}}$ denote the Čech complex of $R$ with respect to $\underline{x}$. The projective system of $R$-modules $\left\{M / \mathfrak{a}^{n} M\right\}_{n \in \mathbb{N}}$ induces a projective system of $R$-complexes

$$
\left\{\check{C}_{\underline{x}} \otimes M / \mathfrak{a}^{n} M\right\} .
$$

2010 AMS Mathematics subject classification. Primary 13D45.

Keywords and phrases. Local cohomology, formal local cohomology.

The first author was partially supported by FAPESP-Brazil-Grants 2012/010840 and 2013/20723-7. The second author was partially supported by CNPq-BrazilGrant 245872/2012-4.

Received by the editors on August 11, 2014, and in revised form on January 31, 2015. 
Consider the projective limit

$$
\lim _{\check{C}}\left(\check{C}_{\underline{x}} \otimes M / \mathfrak{a}^{n} M\right) \text {. }
$$

For an integer $i \in \mathbb{Z}$, the cohomology module

$$
H^{i}\left(\lim _{\longleftarrow}\left(\check{C}_{\underline{x}} \otimes M / \mathfrak{a}^{n} M\right)\right)
$$

is called the $i$ th $\mathfrak{a}$-formal local cohomology with respect to $\mathfrak{b}$, denoted by $\check{\mathfrak{F}}_{\mathfrak{a}, \mathfrak{b}}^{i}(M)$. In the case of $\mathfrak{b}=\mathfrak{m}$, we simply say the $i$ th $\mathfrak{a}$-formal local cohomology.

Now, we consider

$$
\left\{H_{\mathfrak{b}}^{i}\left(M / \mathfrak{a}^{n} M\right)\right\}_{n \in \mathbb{N}},
$$

the family of local cohomology modules. For every integer $n$, there is a natural homomorphism

$$
H_{\mathfrak{b}}^{i}\left(M / \mathfrak{a}^{n+1} M\right) \rightarrow H_{\mathfrak{b}}^{i}\left(M / \mathfrak{a}^{n} M\right)
$$

such that the family forms a projective system. Its projective limit is

$$
\mathfrak{F}_{\mathfrak{a}, \mathfrak{b}}^{i}(M)=\lim _{\longleftarrow} H_{\mathfrak{b}}^{i}\left(M / \mathfrak{a}^{n} M\right) .
$$

When $\mathfrak{b}=\mathfrak{m}$, Schenzel [19] has shown the following isomorphism $\check{\mathfrak{F}}_{\mathfrak{a}, \mathfrak{m}}^{i}(M) \cong \mathfrak{F}_{\mathfrak{a}, \mathfrak{m}}^{i}(M)$, giving the relation between formal local cohomology and projective limits of some local cohomology modules. Also, another approach was studied by Peskine and Szpiro [18, Chapter III] and by Faltings [9].

The main subject of this paper is to generalize the concept of formal local cohomology introduced by Schenzel. In order to do this we will need to use the notion of the local cohomology module with respect to a pair of ideals. That notion was introduced by Takahashi, Yoshino and Yoshizawa [23]. More accurately, let the set of elements of $M$,

$$
\Gamma_{I, J}(M)=\left\{x \in M \mid I^{n} x \subseteq J x \text { for } n \gg 1\right\} .
$$

The functor $\Gamma_{I, J}(-)$ is a left exact functor, additive and covariant on the category of all $R$-modules, called the $(I, J)$-torsion functor. For an integer $i$, the $i$ th right derived functor of $\Gamma_{I, J}(-)$ is denoted by $H_{I, J}^{i}(-)$, and will be referred to as the $i$ th local cohomology functor with respect to $(I, J)$. For an $R$-module $M$, we will refer to $H_{I, J}^{i}(M)$ as the $i$ th local cohomology module of $M$ with respect to $(I, J)$ and $\Gamma_{I, J}(M)$ as the $(I, J)$-torsion part of $M$. When $J=0$ or $J$ is a nilpotent ideal, 
$H_{I, J}^{i}(-)$ coincides with the ordinary local cohomology functor $H_{I}^{i}(-)$ with support in the closed subset $V(I)$.

In [23], the authors have introduced a generalization of Čech complexes as follows. For an element, $x \in R$, let $S_{x, J}$ be the multiplicatively closed subset of $R$ consisting of all elements of the form $x^{n}+j$, where $j \in J$ and $n \in \mathbb{N}$. For an $R$-module $M$, denote by $M_{x, J}=S_{x, J}^{-1} M$. The complex $\check{C}_{x, J}$ is defined as:

$$
\check{C}_{x, J}: 0 \longrightarrow R \longrightarrow R_{x, J} \longrightarrow 0,
$$

where $R$ is sitting in the 0th position, and $R_{x, J}$ in the first position in the complex. For a sequence $\underline{x}=x_{1}, \ldots, x_{s}$ of elements of $R$, let the complex

$$
\check{C}_{\underline{x}, J}=\bigotimes_{i=1}^{s} \check{C}_{x_{i}, J}
$$

If $J=0$, this definition coincides with the usual Cech complex with respect to $\underline{x}=x_{1}, \ldots, x_{s}$.

The organization of the present paper is as follows. In the next section, we will set the formal local cohomology with respect to a pair of ideals and study some elementary properties about this new concept. Conversely to the isomorphism,

$$
\check{\mathfrak{F}}_{\mathfrak{a}, \mathfrak{m}}^{i}(M) \cong \mathfrak{F}_{\mathfrak{a}, \mathfrak{m}}^{i}(M),
$$

commented on above, in our case this does not always happen. We also show in this section some conditions for this isomorphism.

After discussing basic properties of the new concept, in Section 3, we will use the definition of cohomological dimension of $R$-module with respect to a pair of ideals, given by Chu and Wang [5], for establishing some results. We show some exact sequences involving formal local cohomology with respect to a pair of ideals.

In Section 4, we discuss the vanishing and non-vanishing of formal local cohomology with respect to a pair of ideals. In this section, the main result (Theorem 4.2) says that equality

$$
\operatorname{dim}_{R} M /(\mathfrak{a}+J) M=\sup \left\{i \in \mathbb{Z} \mid \mathfrak{F}_{\mathfrak{a}, I, J}^{i}(M) \neq 0\right\},
$$

for ideals $I$ and $J$ of $R$ exists such that $J \neq R$ and the sum of $I+J$ is a m-primary ideal. 
In Section 5, we give a new version of the Mayer-Vietoris sequence for formal local cohomology with respect to a pair of ideals that generalizes [19, Theorem 5.1].

In Section 6, we are interested in showing the relation between formal local cohomology with respect to a pair of ideals and the Matlis dual of certain generalized local cohomology. For this purpose, we show a generalization of the Grothendieck duality theorem for Gorenstein rings, and we obtain another proof of generalized version of the usual local duality theorem for Gorenstein and Cohen-Macaulay rings.

In the last section, we define the concept of formal grade with respect to a pair of ideals and give some results about this.

2. Definition and some results. Let $(R, \mathfrak{m})$ be a local Noetherian ring. Let

$$
\underline{x}=x_{1}, \ldots, x_{s}
$$

be a system of elements of $R$, and $I=(\underline{x}), J$ two ideals of $R$. Let $\check{C}_{\underline{x}, J}$ be the Cech complex of $R$ with respect to $(I, J)$. For an $R$-module $M$ finitely generated and an ideal $\mathfrak{a}$ the projective system of $R$-modules $\left\{M / \mathfrak{a}^{n} M\right\}_{n \in \mathbb{N}}$ induces a projective system of $R$-complexes

$$
\left\{\check{C}_{\underline{x}, J} \otimes M / \mathfrak{a}^{n} M\right\} .
$$

Consider the projective limit

$$
\lim _{\longleftarrow}\left(\check{C}_{\underline{x}, J} \otimes M / \mathfrak{a}^{n} M\right) .
$$

Now, we are able to introduce a new object of study.

Definition 2.1. Using the previous construction, for an integer $i \in \mathbb{Z}$, the cohomology module

$$
H^{i}\left(\varliminf_{\longleftarrow}\left(\check{C}_{\underline{x}, J} \otimes M / \mathfrak{a}^{n} M\right)\right)
$$

is called the Cech $i$ th $\mathfrak{a}$-formal cohomology with respect to $(I, J)$, denoted by $\check{\mathfrak{F}}_{\mathfrak{a}, I, J}^{i}(M)$.

Note that, if $J=0$, then $\check{C}_{\underline{x}, J}$ coincides with the usual Čech complex $\check{C}_{\underline{x}}$ with respect to $\underline{x}=x_{1}, \ldots, x_{s}$. In this case,

$$
\check{\mathfrak{F}}_{\mathfrak{a}, I, 0}^{i}(M) \cong \check{\mathfrak{F}}_{\mathfrak{a}, I}^{i}(M) \text {. }
$$


If $J=0$ and $I=\mathfrak{m}$, we have

$$
\check{\mathfrak{F}}_{\mathfrak{a}, \mathfrak{m}, 0}^{i}(M) \cong \check{\mathfrak{F}}_{\mathfrak{a}, \mathfrak{m}}^{i}(M) .
$$

This definition is a natural generalization of $\mathfrak{a}$-formal cohomology with respect to $\mathfrak{b}$ and $\mathfrak{a}$-formal cohomology, both introduced by Schenzel in [19], and discussed by Mafi [17], Asgharzadeh and Divaaani-Aazar [2] and Eghbali [8].

Proposition 2.2. Let $M$ be a finitely generated $R$-module. If $M$ is a $J$-torsion $R$-module, then

$$
\check{\mathfrak{F}}_{\mathfrak{a}, I, J}^{i}(M) \cong \check{\mathfrak{F}}_{\mathfrak{a}, I}^{i}(M) .
$$

Proof. By [23, Corollary 2.5], we have that

$$
\check{C}_{\underline{x}, J} \otimes M / \mathfrak{a}^{n} M \cong \check{C}_{\underline{x}} \otimes M / \mathfrak{a}^{n} M .
$$

Applying the inverse limit, we obtain

$$
\lim _{\longleftarrow}\left(\check{C}_{\underline{x}, J} \otimes M / \mathfrak{a}^{n} M\right) \cong \lim _{\longleftarrow}\left(\check{C}_{\underline{x}} \otimes M / \mathfrak{a}^{n} M\right) .
$$

Therefore,

$\check{\mathfrak{F}}_{\mathfrak{a}, I, J}^{i}(M)=H^{i}\left(\lim _{\longleftarrow}\left(\check{C}_{\underline{x}, J} \otimes M / \mathfrak{a}^{n} M\right)\right)=H^{i}\left(\lim _{\longleftarrow}\left(\check{C}_{\underline{x}} \otimes M / \mathfrak{a}^{n} M\right)\right)=\check{\mathfrak{F}}_{\mathfrak{a}, I}^{i}(M)$.

The following theorem is a generalization of the base ring independence theorem for formal local cohomology and local cohomology modules. The reader can compare this result with [2, Lemma 2.1 (a)], [4, Theorem 4.2.1] and [23, Theorem 2.7].

Theorem 2.3. Let $\phi: R \rightarrow R^{\prime}$ be a ring homomorphism such that $\phi(J)=J R^{\prime}$, and let $M^{\prime}$ be a finitely generated $R^{\prime}$-module. Then there is a natural isomorphism,

$$
\check{\mathfrak{F}}_{\mathfrak{a}, I, J}^{i}\left(M^{\prime}\right) \cong \check{\mathfrak{F}}_{\mathfrak{a} R^{\prime}, I R^{\prime}, J R^{\prime}}^{i}\left(M^{\prime}\right)
$$

for all $i \in \mathbb{N}_{0}$.

Proof. Set

$$
I=\left(x_{1}, \ldots, x_{s}\right) R
$$


and

$$
\phi(\underline{x})=\phi\left(x_{1}\right), \ldots, \phi\left(x_{s}\right) .
$$

Let $S_{x_{i}, J}$ be a multiplicatively closed subset of $R$. By hypothesis, $\phi\left(S_{x_{i}, J}\right)=S_{\phi\left(x_{i}\right), J R^{\prime}}$, for all $i$ with $1 \leq i \leq s$. Using the map $\phi$, we can also regard $M^{\prime}$ as an $R$-module, and thus the Cech complex $\check{C}_{\underline{x}, J}$ is the same whether we regard $M^{\prime}$ as an $R$-module or an $R^{\prime}$-module. Therefore, we have

$$
\begin{aligned}
\check{C}_{\underline{x}, J} \otimes_{R} M^{\prime} / \mathfrak{a}^{n} M^{\prime} & \cong \check{C}_{\underline{x}, J} \otimes_{R}\left(R^{\prime} \otimes_{R^{\prime}} M^{\prime} / \mathfrak{a}^{n} M^{\prime}\right) \\
& \cong\left(\check{C}_{\underline{x}, J} \otimes_{R} R^{\prime}\right) \otimes_{R^{\prime}} M^{\prime} / \mathfrak{a}^{n} M^{\prime} \\
& \cong \check{C}_{\phi(\underline{x}), J R^{\prime}} \otimes_{R^{\prime}} M^{\prime} / \mathfrak{a} R^{\prime n} M^{\prime} .
\end{aligned}
$$

Applying the inverse limit and Definition 2.1 gives the statement.

It is important to remember that the hypothesis $\phi(J)=J R^{\prime}$ in the previous theorem cannot be removed. For more details, see [23, Remark 2.8].

Now, consider

$$
\left\{H_{I, J}^{i}\left(M / \mathfrak{a}^{n} M\right)\right\}_{n \in \mathbb{N}}
$$

to be the family of local cohomology modules. There is a natural homomorphism

$$
H_{I, J}^{i}\left(M / \mathfrak{a}^{n+1} M\right) \rightarrow H_{I, J}^{i}\left(M / \mathfrak{a}^{n} M\right),
$$

for all $n$, such that the family forms a projective system. Their projective limit

$$
\lim _{\longleftarrow} H_{I, J}^{i}\left(M / \mathfrak{a}^{n} M\right)
$$

is called the $i$ th formal local cohomology of $M$ with respect to a pair ideals, $I$ and $J$, and we denote $\mathfrak{F}_{\mathfrak{a}, I, J}^{i}(M)$. In the cases $I=\mathfrak{m}$ and $J=0$, Schenzel [19, Proposition 3.2] showed that

$$
\breve{\mathfrak{F}}_{\mathfrak{a}, \mathfrak{m}, 0}^{i}(M) \cong \mathfrak{F}_{\mathfrak{a}, \mathfrak{m}, 0}^{i}(M) .
$$

The natural question arises: when is $\breve{\mathfrak{F}}_{\mathfrak{a}, I, J}^{i}(M)$ isomorphic to $\mathfrak{F}_{\mathfrak{a}, I, J}^{i}(M)$ ? The next result improves [19, Proposition 3.2] and tries to answer this question. 
Proposition 2.4. Using the previous notation, there is the short exact sequence

$0 \longrightarrow \lim _{\leftarrow}^{1} H_{I, J}^{i-1}\left(M / \mathfrak{a}^{n} M\right) \longrightarrow H^{i}\left(\lim _{\leftarrow}\left(\check{C}_{\underline{x}, J} \otimes M / \mathfrak{a}^{n} M\right)\right) \longrightarrow \lim _{\leftarrow} H_{I, J}^{i}\left(M / \mathfrak{a}^{n} M\right) \longrightarrow 0$ for all $i \in \mathbb{Z}$.

Proof. For $n \in \mathbb{N}$, let the natural epimorphism be $M / \mathfrak{a}^{n+1} M \rightarrow$ $M / \mathfrak{a}^{n} M$. Since the Čech complex $\check{C}_{\underline{x}, J}$ is a complex of flat $R$-modules, we have an $R$-morphism of $R$-complexes

$$
\check{C}_{\underline{x}, J} \otimes M / \mathfrak{a}^{n+1} M \rightarrow \check{C}_{\underline{x}, J} \otimes M / \mathfrak{a}^{n} M
$$

(which is degree-wise an epimorphism). By [24, Definition 3.5.1 and Lemma 3.5.3], it follows that the short exact sequence of complexes

$0 \longrightarrow \lim _{\leftarrow}\left(\check{C}_{\underline{x}, J} \otimes M / \mathfrak{a}^{n} M\right) \longrightarrow \prod\left(\check{C}_{\underline{x}, J} \otimes M / \mathfrak{a}^{n} M\right) \longrightarrow \prod\left(\check{C}_{\underline{x}, J} \otimes M / \mathfrak{a}^{n} M\right) \longrightarrow 0$.

So, there is the long exact cohomology sequence,

$$
\cdots \longrightarrow H^{i}\left(\lim _{\longleftarrow}\left(\check{C}_{\underline{x}, J} \otimes M / \mathfrak{a}^{n} M\right)\right) \longrightarrow H^{i}\left(K^{\bullet}\right) \stackrel{\phi^{i}}{\longrightarrow} H^{i}\left(K^{\bullet}\right) \longrightarrow \cdots,
$$

where

$$
K^{\bullet}=\prod\left(\check{C}_{\underline{x}, J} \otimes M / \mathfrak{a}^{n} M\right) .
$$

Since the cohomology commutes with direct products we obtain a morphism

$$
\phi^{i}: \prod H^{i}\left(\check{C}_{\underline{x}, J} \otimes M / \mathfrak{a}^{n} M\right) \longrightarrow \prod H^{i}\left(\check{C}_{\underline{x}, J} \otimes M / \mathfrak{a}^{n} M\right),
$$

where the map is induced by the transition map of inverse systems of the complexes. Now it is known that the kernel of $\phi^{i}$ and cokernel of $\phi^{i-1}$ are, respectively,

$$
\lim _{\longleftarrow} H_{I, J}^{i}\left(M / \mathfrak{a}^{n} M\right)
$$

and

$$
\varliminf_{\lim ^{1}} H_{I, J}^{i-1}\left(M / \mathfrak{a}^{n} M\right)
$$

[24, Definition 3.5.1]. This finishes the proof.

To answer the previous question, we need to know when the local cohomology with respect to a pair of ideals is an Artinian $R$-module for all $i \in \mathbb{Z}$. Recently, Tehranian and Talemi [22, Theorem 2.10] have shown that, for $J$ to be a non-nilpotent ideal of a local ring $(R, \mathfrak{m})$ and 
$M$ to be a finite $R$-module, $H_{\mathfrak{m}, J}^{i}(M)$ is not Artinian for some positive integer $i$. We show below some cases where local cohomology defined by a pair of ideals is Artinian.

Remark 2.5. Let $M$ be a finitely generated $R$-module.

(a) If $M$ is a $J$-torsion $R$-module, then $H_{\mathfrak{m}, J}^{i}(M)$ is an Artinian $R$ module for all integers $i$, because

$$
H_{\mathfrak{m}, J}^{i}(M) \cong H_{\mathfrak{m}}^{i}(M)
$$

[4, Theorem 7.1.3] and [23, Corollary 2.5].

(b) If $M$ is a $J$-torsion $R$-module and $\sqrt{I+J}=\mathfrak{m}$, then $H_{I, J}^{i}(M)$ is an Artinian $R$-module for all integers $i$. It follows by [23, Proposition $1.4(6),(7)]$ that

$$
H_{I, J}^{i}(M)=H_{\sqrt{I+J}, J}^{i}(M)=H_{\mathfrak{m}, J}^{i}(M)=H_{\mathfrak{m}}^{i}(M),
$$

which is an Artinian $R$-module.

Now, we are able to give some conditions for the isomorphism between formal local cohomology and Čech formal local cohomology with respect to a pair of ideals.

Corollary 2.6. Let $M$ be a finitely generated $R$-module. The following hold.

(i)

$$
\check{\mathfrak{F}}_{\mathfrak{a}, \mathfrak{m}, J}^{i}(M)=\mathfrak{F}_{\mathfrak{a}, \mathfrak{m}, J}^{i}(M)
$$

for all $i \in \mathbb{Z}$, if $M$ is a $J$-torsion $R$-module.

(ii)

$$
\check{\mathfrak{F}}_{\mathfrak{a}, I, J}^{i}(M)=\mathfrak{F}_{\mathfrak{a}, I, J}^{i}(M)
$$

for all $i \in \mathbb{Z}$, if $M$ is a $J$-torsion $R$-module and $\sqrt{I+J}=\mathfrak{m}$.

(iii) If $M$ is an Artinian $R$-module, then

$$
\check{\mathfrak{F}}_{\mathfrak{a}, I, J}^{i}(M)=\mathfrak{F}_{\mathfrak{a}, I, J}^{i}(M)
$$

for all $i \in \mathbb{Z}$.

Proof. To prove statement (i), note that $M / \mathfrak{a}^{n} M$ is a $J$-torsion $R$ module. Then, by the previous remark, $H_{\mathfrak{m}, J}^{i}\left(M / \mathfrak{a}^{n} M\right)$ is an Artinian 
$R$-module for all $i \in \mathbb{Z}$. So the family

$$
\left\{H_{\mathfrak{m}, J}^{i}\left(M / \mathfrak{a}^{n} M\right)\right\}_{n \in \mathbb{Z}}, \quad i \in \mathbb{N},
$$

satisfies the Mittag-Leffler condition. By Proposition 2.4 and using the fact that $\lim ^{1}$ vanishes on the projective system of Artinian $R$-modules, the statement follows.

The proof of (ii) is analogous.

For (iii), use the previous remark to show that $H_{I, J}^{i}\left(M / \mathfrak{a}^{n} M\right)$ is an Artinian $R$-module, for all $i \in \mathbb{Z}$. Applying the previous idea finishes the proof.

Corollary 2.7. Let $\phi: R \rightarrow R^{\prime}$ be a ring homomorphism such that $\phi(J)=J R^{\prime}$, and let $M^{\prime}$ be a finitely generated $R^{\prime}$-module.

$$
\mathfrak{F}_{\mathfrak{a}, \mathfrak{m}, J}^{i}\left(M^{\prime}\right)=\mathfrak{F}_{\mathfrak{a} R^{\prime}, \mathfrak{m} R^{\prime}, J R^{\prime}}^{i}\left(M^{\prime}\right)
$$

for all $i \in \mathbb{Z}$, if $M^{\prime}$ is a J-torsion $R^{\prime}$-module.

$$
\mathfrak{F}_{\mathfrak{a}, I, J}^{i}\left(M^{\prime}\right)=\mathfrak{F}_{\mathfrak{a} R^{\prime}, I R^{\prime}, J R^{\prime}}^{i}\left(M^{\prime}\right)
$$

for all $i \in \mathbb{Z}$, if $M^{\prime}$ is a J-torsion $R^{\prime}$-module and $\sqrt{I+J}=\mathfrak{m}$.

$$
\mathfrak{F}_{\mathfrak{a}, I, J}^{i}\left(M^{\prime}\right)=\mathfrak{F}_{\mathfrak{a} R^{\prime}, I R^{\prime}, J R^{\prime}}^{i}\left(M^{\prime}\right)
$$

for all $i \in \mathbb{Z}$, if $M^{\prime}$ is an Artinian $R^{\prime}$-module.

Proof. Since $M^{\prime}$ is $J R^{\prime}$-torsion, too, by Corollary 2.7 and Theorem 2.3, we have the proof of (i). The same idea can be used in assertions (ii) and (iii).

3. Cohomological dimension. In this section, we study some preliminary results on cohomological dimension of the $R$-module $M$ with respect to a pair of ideals $(I, J)$, and we give some exact sequences involving formal cohomology with respect to a pair of ideals. Firstly, it is known that Divaani-Aazar, Naghipour and Tousi [6] were the precursors on the term cohomological dimension, defined by

$$
\operatorname{cd}(\mathfrak{a}, M)=\sup \left\{i \in \mathbb{Z}: H_{\mathfrak{a}}^{i}(M) \neq 0\right\} .
$$


Referring to local cohomology defined by a pair of ideals, Chu and Wang [5] defined the cohomological dimension of the $R$-module $M$ with respect to a pair of ideals, $(I, J)$, given by

$$
\operatorname{cd}(I, J, M)=\sup \left\{i \in \mathbb{Z}: H_{I, J}^{i}(M) \neq 0\right\},
$$

and showed a characterization of this integer.

Chu and Wang [5] also generalized the result of Schenzel [19, Lemma 2.1] using this new concept. This result is given in the following proposition.

Proposition 3.1. Let I be a proper ideal of a commutative Noetherian ring $R$, and let $M$ and $N$ be a finitely generated pair of $R$-modules such that $\operatorname{Supp}_{R} N \subseteq \operatorname{Supp}_{R} M$. Then $\operatorname{cd}(I, J, N) \leq \operatorname{cd}(I, J, M)$.

Corollary 3.2. Let $M$ be a finitely generated $R$-module. Then

$$
\operatorname{cd}(I, J, M)=\max \{\operatorname{cd}(I, J, R / \mathfrak{p}): \mathfrak{p} \in \operatorname{Min} M\} .
$$

Proof. The proof is similar to [19, Corollary 2.2].

Lemma 3.3. Let $\underline{x}=x_{1}, \ldots, x_{s}$ be a system of elements of the ring $R, \mathfrak{a}=(\underline{x})$ the ideal of $R$, and let $M$ be a finitely generated $R$-module. Then

$$
\operatorname{cd}((\mathfrak{a}, y R), J, M) \leq \operatorname{cd}(\mathfrak{a}, J, M)+1
$$

for any element $y \in \mathfrak{m}$.

Proof. The proof of this statement follows by replacing the Čech complex by the extended Čech complex of [23] in [19, Lemma 2.3].

These two previous results extend [19, Corollary 2.2, Lemma 2.3]. Now, we will analyze how the Cech formal local cohomology with respect to a pair of ideals behaves with regard to short exact sequences. In order to prove the next result we employ an argument analogue to that used by Schenzel in [19, Remark 3.12].

Theorem 3.4. Let

$$
0 \longrightarrow A \longrightarrow B \longrightarrow C \longrightarrow 0
$$


denote a short exact sequence of finitely generated $R$-modules. Then there is a long exact sequence,

$$
\cdots \longrightarrow \check{\mathfrak{F}}_{\mathfrak{a}, I, J}^{i}(A) \longrightarrow \check{\mathfrak{F}}_{\mathfrak{a}, I, J}^{i}(B) \longrightarrow \check{\mathfrak{F}}_{\mathfrak{a}, I, J}^{i}(C) \longrightarrow \check{\mathfrak{F}}_{\mathfrak{a}, I, J}^{i+1}(A) \longrightarrow \cdots
$$

Proof. It is well known that the previous short exact sequence induces a projective system of short exact sequences:

$0 \longrightarrow \check{C}_{\underline{x}, J} \otimes A / B \cap \mathfrak{a}^{n} A \longrightarrow \check{C}_{\underline{x}, J} \otimes B / \mathfrak{a}^{n} B \longrightarrow \check{C}_{\underline{x}, J} \otimes C / \mathfrak{a}^{n} C \longrightarrow 0$

for all $n \in \mathbb{N}$. Because $\check{C}_{\underline{x}, J}$ is a complex of flat $R$-modules and the maps

$$
A / B \cap \mathfrak{a}^{n+1} A \longrightarrow A / B \cap \mathfrak{a}^{n} A
$$

are surjective, it follows that the projective system of $R$-complexes $\left\{\check{C}_{\underline{x}, J} \otimes A / B \cap \mathfrak{a}^{n} A\right\}$ satisfies the Mittag-Leffler condition. Therefore, by [11, Proposition 13.2.2], we have the exact sequence of complexes

$$
\begin{aligned}
& 0 \longrightarrow \lim _{\longleftarrow} \check{C}_{\underline{x}, J} \otimes A / B \cap \mathfrak{a}^{n} A \longrightarrow \lim _{\longleftarrow} \check{C}_{\underline{x}, J} \otimes B / \mathfrak{a}^{n} B \\
& \longrightarrow \lim _{\longleftarrow} \check{C}_{\underline{x}, J} \otimes C / \mathfrak{a}^{n} C \longrightarrow 0 .
\end{aligned}
$$

In the case where $\left\{B \cap \mathfrak{a}^{n} A\right\}$ is equivalent to the $\mathfrak{a}$-adic topology on $A$ and by the Artin-Rees lemma [3, Chapter III, Section 3, Corollary 1], we have

$$
\begin{aligned}
\cdots \longrightarrow H^{i}\left(\lim _{\longleftarrow} \check{C}_{\underline{x}, J} \otimes A / \mathfrak{a}^{n} A\right) & \longrightarrow H^{i}\left(\lim _{\longleftarrow} \check{C}_{\underline{x}, J} \otimes B / \mathfrak{a}^{n} B\right) \\
& \longrightarrow H^{i}\left(\varliminf_{\longleftarrow} \check{C}_{\underline{x}, J} \otimes C / \mathfrak{a}^{n} C\right) \longrightarrow \cdots .
\end{aligned}
$$

Using the definition of Cech formal local cohomology defined by a pair of ideals finishes the proof.

Corollary 3.5. Using the same hypothesis as the previous theorem, there is the long exact sequence:

(i) If $B$ is a J-torsion $R$-module, then

$$
\cdots \longrightarrow \mathfrak{F}_{\mathfrak{a}, \mathfrak{m}, J}^{i}(A) \longrightarrow \mathfrak{F}_{\mathfrak{a}, \mathfrak{m}, J}^{i}(B) \longrightarrow \mathfrak{F}_{\mathfrak{a}, \mathfrak{m}, J}^{i}(C) \longrightarrow \mathfrak{F}_{\mathfrak{a}, \mathfrak{m}, J}^{i+1}(A) \longrightarrow \cdots
$$

(ii) If $B$ is a J-torsion R-module and $\sqrt{I+J}=\mathfrak{m}$, then

$$
\cdots \longrightarrow \mathfrak{F}_{\mathfrak{a}, I, J}^{i}(A) \longrightarrow \mathfrak{F}_{\mathfrak{a}, I, J}^{i}(B) \longrightarrow \mathfrak{F}_{\mathfrak{a}, I, J}^{i}(C) \longrightarrow \mathfrak{F}_{\mathfrak{a}, I, J}^{i+1}(A) \longrightarrow \cdots
$$


(iii) If $B$ is an Artinian $R$-module, then

$\cdots \longrightarrow \mathfrak{F}_{\mathfrak{a}, I, J}^{i}(A) \longrightarrow \mathfrak{F}_{\mathfrak{a}, I, J}^{i}(B) \longrightarrow \mathfrak{F}_{\mathfrak{a}, I, J}^{i}(C) \longrightarrow \mathfrak{F}_{\mathfrak{a}, I, J}^{i+1}(A) \longrightarrow \cdots$

Proof. To prove all cases, apply Corollary 2.6 and Theorem 3.4.

Our next result can be considered as a slight extension of $[\mathbf{1 9}$, Corollary 3.13].

Proposition 3.6. Let $M$ be a finitely generated $R$-module, $N \subseteq M$ an $R$-module such that $\operatorname{Supp} N \cap V(\mathfrak{a}) \subseteq V(\mathfrak{m})$ and $\bar{M}=M / N$. Then there is a short exact sequence:

$$
0 \longrightarrow N^{\mathfrak{a}} \longrightarrow \mathfrak{F}_{\mathfrak{a}, I, J}^{0}(M) \longrightarrow \mathfrak{F}_{\mathfrak{a}, I, J}^{0}(\bar{M}) \longrightarrow 0
$$

and isomorphisms,

$$
\mathfrak{F}_{\mathfrak{a}, I, J}^{i}(M) \cong \mathfrak{F}_{\mathfrak{a}, I, J}^{i}(\bar{M})
$$

for all $i \geq 1$.

Proof. Consider the short exact sequence

$$
0 \longrightarrow N \longrightarrow M \longrightarrow \bar{M} \longrightarrow 0 \text {. }
$$

As in Theorem 3.4, there is the following long exact sequence,

$$
0 \longrightarrow \check{C}_{\underline{x}, J} \otimes N / \mathfrak{a}^{n} N \longrightarrow \check{C}_{\underline{x}, J} \otimes M / \mathfrak{a}^{n} M \longrightarrow \check{C}_{\underline{x}, J} \otimes \bar{M} / \mathfrak{a}^{n} \bar{M} \longrightarrow 0,
$$

for all $n \in \mathbb{N}$. This sequence induces a long exact cohomology sequence $\cdots \longrightarrow H^{i}\left(\check{C}_{\underline{x}, J} \otimes N / \mathfrak{a}^{n} N\right) \longrightarrow H^{i}\left(\check{C}_{\underline{x}, J} \otimes M / \mathfrak{a}^{n} M\right) \longrightarrow H^{i}\left(\check{C}_{\underline{x}, J} \otimes \bar{M} / \mathfrak{a}^{n} \bar{M}\right) \longrightarrow \cdots$.

By [23, Theorem 2.4], we have that $H^{i}\left(\check{C}_{\underline{x}, J} \otimes X\right) \cong H_{I, J}^{i}(X)$ for any $R$-module $X$, so we obtain the sequence

$$
\cdots \longrightarrow H_{I, J}^{i}\left(N / \mathfrak{a}^{n} N\right) \longrightarrow H_{I, J}^{i}\left(M / \mathfrak{a}^{n} M\right) \longrightarrow H_{I, J}^{i}\left(\bar{M} / \mathfrak{a}^{n} \bar{M}\right) \longrightarrow \cdots
$$

The assumption $\operatorname{Supp} N \cap V(\mathfrak{a}) \subseteq V(\mathfrak{m})$ implies that $N / \mathfrak{a}^{n} N$ is an $R$-module of finite length, for all $n \in \mathbb{N}$. By [23, Theorem 4.7], $H_{I, J}^{i}\left(N / \mathfrak{a}^{n} N\right)=0$ for all $i>0$. Therefore, we have

$$
0 \longrightarrow H_{I, J}^{0}\left(N / \mathfrak{a}^{n} N\right) \longrightarrow H_{I, J}^{0}\left(M / \mathfrak{a}^{n} M\right) \longrightarrow H_{I, J}^{0}\left(\bar{M} / \mathfrak{a}^{n} \bar{M}\right) \longrightarrow 0,
$$

and isomorphisms,

$$
H_{I, J}^{i}\left(M / \mathfrak{a}^{n} M\right) \cong H_{I, J}^{i}\left(\bar{M} / \mathfrak{a}^{n} \bar{M}\right) \text { for all } i>0 .
$$


Note that the family of Artinian $R$-modules $\left\{H_{I, J}^{0}\left(N / \mathfrak{a}^{n} N\right)\right\}_{n \in \mathbb{N}}([\mathbf{2 3}$, Theorem 4.7 and Corollary 4.2] shows that $H_{I, J}^{0}\left(N / \mathfrak{a}^{n} N\right)$ is Artinian), satisfies the Mittag-Leffler condition. Passing to the projective limit in the previous exact sequence we obtain

$$
0 \longrightarrow \mathfrak{F}_{\mathfrak{a}, I, J}^{0}(N) \longrightarrow \mathfrak{F}_{\mathfrak{a}, I, J}^{0}(M) \longrightarrow \mathfrak{F}_{\mathfrak{a}, I, J}^{0}(\bar{M}) \longrightarrow 0,
$$

and isomorphisms

$$
\mathfrak{F}_{\mathfrak{a}, I, J}^{i}(M) \cong \mathfrak{F}_{\mathfrak{a}, I, J}^{i}(\bar{M}) \text { for all } i \geq 1 .
$$

Now, as for all $i>0, H_{I, J}^{i}\left(N / \mathfrak{a}^{n} N\right)=0$, by [23, Corollary 4.2], it follows that $M$ is an $(I, J)$-torsion $R$-module. Therefore,

$$
H_{I, J}^{0}\left(N / \mathfrak{a}^{n} N\right)=N / \mathfrak{a}^{n} N
$$

and

$$
\mathfrak{F}_{\mathfrak{a}, I, J}^{0}(N)=\lim _{\longleftarrow} N / \mathfrak{a}^{n} N=N^{\mathfrak{a}} .
$$

Corollary 3.7. Consider the same hypothesis as in Proposition 3.6.

(i) If $M$ is a $J$-torsion $R$-module, then there is a short exact sequence,

$$
0 \longrightarrow \check{\mathfrak{F}}_{\mathfrak{a}, \mathfrak{m}, J}^{0}(N) \longrightarrow \check{\mathfrak{F}}_{\mathfrak{a}, \mathfrak{m}, J}^{0}(M) \longrightarrow \check{\mathfrak{F}}_{\mathfrak{a}, \mathfrak{m}, J}^{0}(\bar{M}) \longrightarrow 0,
$$

and isomorphisms,

$$
\check{\mathfrak{F}}_{\mathfrak{a}, \mathfrak{m}, J}^{i}(M) \cong \check{\mathfrak{F}}_{\mathfrak{a}, \mathfrak{m}, J}^{i}(\bar{M})=0 \quad \text { for all } i \geq 1 .
$$

(ii) If $M$ is a $J$-torsion $R$-module and $\sqrt{I+J}=\mathfrak{m}$, then there is a short exact sequence,

$$
0 \longrightarrow \check{\mathfrak{F}}_{\mathfrak{a}, I, J}^{0}(N) \longrightarrow \check{\mathfrak{F}}_{\mathfrak{a}, I, J}^{0}(M) \longrightarrow \check{\mathfrak{F}}_{\mathfrak{a}, I, J}^{0}(\bar{M}) \longrightarrow 0,
$$

and isomorphisms,

$$
\breve{\mathfrak{F}}_{\mathfrak{a}, I, J}^{i}(M) \cong \check{\mathfrak{F}}_{\mathfrak{a}, I, J}^{i}(\bar{M})=0 \quad \text { for all } i \geq 1 .
$$

Proof. Use Corollary 2.6 and Proposition 3.6.

Theorem 3.8. For a finitely generated $R$-module $M$, choose an element $x \in \mathfrak{m}$ such that $x \notin \mathfrak{p}$, for all $\mathfrak{p} \in A s s_{R} M \backslash\{\mathfrak{m}\}$. Let $M^{\prime}=M / x M$. 
(i) If $M$ is a J-torsion $R$-module, then, for all $i \in \mathbb{Z}$, there is a short exact sequence:

$0 \longrightarrow H_{0}\left(x ; \mathfrak{F}_{\mathfrak{a}, \mathfrak{m}, J}^{i}(M)\right) \longrightarrow \lim _{\longleftarrow} H_{\mathfrak{m}, J}^{i}\left(M^{\prime} / \mathfrak{a}^{n} M^{\prime}\right) \longrightarrow H_{1}\left(x ; \mathfrak{F}_{\mathfrak{a}, \mathfrak{m}, J}^{i+1}(M)\right) \longrightarrow 0$.

(ii) If $M$ is a J-torsion R-module and $\sqrt{I+J}=\mathfrak{m}$, then, for all $i \in \mathbb{Z}$, there is a short exact sequence:

$0 \longrightarrow H_{0}\left(x ; \mathfrak{F}_{\mathfrak{a}, I, J}^{i}(M)\right) \longrightarrow \lim _{\longleftarrow} H_{I, J}^{i}\left(M^{\prime} / \mathfrak{a}^{n} M^{\prime}\right) \longrightarrow H_{1}\left(x ; \mathfrak{F}_{\mathfrak{a}, I, J}^{i+1}(M)\right) \longrightarrow 0$.

Proof. The proof of statement (i) is the same as in [19, Theorem 3.14], replacing the usual formal local cohomology by formal local cohomology defined by a pair of ideals. The proof of claim (ii) is analogous.

4. Vanishing and non-vanishing results. In this section, we will discuss vanishing and non-vanishing of the formal local cohomology with respect to a pair of ideals. Let $M$ be a finitely generated $R$ module. Let $\mathfrak{a}, I=(\underline{x}), J$ be ideals of the local ring $(R, \mathfrak{m})$. Our purpose in this section is to describe the integer

$$
\sup \left\{i \in \mathbb{Z} \mid \mathfrak{F}_{\mathfrak{a}, I, J}^{i}(M) \neq 0\right\} .
$$

We begin with a simple but important result that extends [19, Proposition 4.4].

Proposition 4.1. Consider an ideal a such that $\operatorname{dim}(M / \mathfrak{a} M)=0$. Then:

$$
\begin{aligned}
& \mathfrak{F}_{\mathfrak{a}, I, J}^{i}(M)= \begin{cases}0 & \text { if } i \neq 0 \\
M^{\mathfrak{a}} & \text { if } i=0 .\end{cases} \\
& \mathfrak{F}_{\mathfrak{a}, I, J}^{i}(M)=\check{\mathfrak{F}}_{\mathfrak{a}, I, J}^{i}(M), \quad \text { for all } i \in \mathbb{Z} .
\end{aligned}
$$

Proof. For the proof of (i) note that $H_{I, J}^{i}\left(M / \mathfrak{a}^{n} M\right)=0$ for $i \neq 0$ by [23, Theorem 4.7], and $M / \mathfrak{a}^{n} M$ is an $(I, J)$-torsion $R$-module by [23, Corollary 4.2]. Then

$$
H_{I, J}^{0}\left(M / \mathfrak{a}^{n} M\right)=\Gamma_{I, J}\left(M / \mathfrak{a}^{n} M\right)=M / \mathfrak{a}^{n} M .
$$

Passing to the projective limit finishes the proof.

For the proof of (ii) use Proposition 2.4. 
As a brief comment, remember that Grothendieck's non-vanishing theorem says that $H_{\mathfrak{m}}^{r}(M) \neq 0$ when $R$ is a local ring with maximal ideal $\mathfrak{m}$, and $M$ is a finitely generated $R$-module of dimension $r$. Takahashi, Yoshino and Yoshizawa [23, Theorem 4.5] have shown a theorem that could be seen as a generalization of this famous result. Schenzel [19, Theorem 4.5] established a similar result to Grothendieck's nonvanishing theorem for formal local cohomology. The following theorem is a generalization of the result assigned by Schenzel.

Theorem 4.2. Let $M$ be a finitely generated module over a local ring $(R, \mathfrak{m})$. Let $\mathfrak{a}, I, J$ be ideals of $R$ such that $J \neq R$ and $I+J$ is an $\mathfrak{m}$-primary ideal. Then,

$$
\operatorname{dim} M /(\mathfrak{a}+J) M=\sup \left\{i \in \mathbb{Z} \mid \mathfrak{F}_{\mathfrak{a}, I, J}^{i}(M) \neq 0\right\} .
$$

Proof. By [23, Theorem 4.3], we have that $H_{I, J}^{i}\left(M / \mathfrak{a}^{n} M\right)=0$ for all $i>\operatorname{dim}\left(M / \mathfrak{a}^{n} M\right) /\left(J\left(M / \mathfrak{a}^{n} M\right)\right)$. But,

$$
\operatorname{dim} \frac{M / \mathfrak{a}^{n} M}{J\left(M / \mathfrak{a}^{n} M\right)}=\operatorname{dim} \frac{M}{(J+\mathfrak{a}) M} \quad \text { for all } n \in \mathbb{N} .
$$

Therefore,

$$
\operatorname{dim} M /(\mathfrak{a}+J) M \geq \sup \left\{i \in \mathbb{Z} \mid \mathfrak{F}_{\mathfrak{a}, I, J}^{i}(M) \neq 0\right\} .
$$

On the other hand, consider

$$
r:=\operatorname{dim}\left(\frac{M / \mathfrak{a}^{n} M}{J\left(M / \mathfrak{a}^{n} M\right)}\right)=\operatorname{dim}\left(\frac{M}{(J+\mathfrak{a}) M}\right) \text { for all } n \in \mathbb{N} .
$$

Since $I+J$ is an $\mathfrak{m}$-primary ideal, we have $H_{I, J}^{i}(M)=H_{\mathfrak{m}, J}^{i}(M)$ for any integer $i$. Thus, we may assume $I=\mathfrak{m}$. Note that the short exact sequence:

$$
0 \longrightarrow \mathfrak{a}^{n} M / \mathfrak{a}^{n+1} M \longrightarrow M / \mathfrak{a}^{n} M \longrightarrow M / \mathfrak{a}^{n+1} M \longrightarrow 0
$$

induces the long exact sequence

$\cdots \longrightarrow H_{\mathfrak{m}, J}^{i}\left(M / \mathfrak{a}^{n+1} M\right) \longrightarrow H_{\mathfrak{m}, J}^{i}\left(M / \mathfrak{a}^{n+1} M\right) \longrightarrow H_{\mathfrak{m}, J}^{i+1}\left(\mathfrak{a}^{n} M / \mathfrak{a}^{n+1} M\right) \longrightarrow \cdots$

Now denote $\bar{M}=\mathfrak{a}^{n} M / \mathfrak{a}^{n+1} M$. Since

$$
\operatorname{dim} \bar{M} / J \bar{M} \leq \operatorname{dim} \frac{M}{(J+\mathfrak{a}) M}=r,
$$


$H_{\mathfrak{m}, J}^{r+1}(\bar{M})=0$ by [23, Theoren 4.3] and $H_{\mathfrak{m}, J}^{r}\left(M / \mathfrak{a}^{n} M\right) \neq 0$ by [23, Theorem 4.5].

Therefore, we have an epimorphism

$$
H_{\mathfrak{m}, J}^{r}\left(M / \mathfrak{a}^{n+1} M\right) \longrightarrow H_{\mathfrak{m}, J}^{r}\left(M / \mathfrak{a}^{n} M\right) \longrightarrow 0
$$

of non-zero $R$-modules for all $n \in \mathbb{N}$. This implies that $\mathfrak{F}_{\mathfrak{a}, I, J}^{r}(M) \neq 0$.

Remark 4.3. Let $M$ be a finitely generated $R$-module. Some properties follow about vanishing of the formal local cohomology with respect to a pair of ideals:

(a) $\mathfrak{F}_{\mathfrak{a}, I, J}^{i}(M)=0$ for any $i>\operatorname{dim}(M / \mathfrak{a} M)$ [23, Theorem 4.7].

(b) $\mathfrak{F}_{\mathfrak{a}, I, J}^{i}(M)=0$ for any $i>\operatorname{dim}(M /(\mathfrak{a}+J) M)$, if $J \neq R[\mathbf{2 3}$, Theorem 4.3].

(c) $\mathfrak{F}_{\mathfrak{a}, I, J}^{i}(M)=0$ for any $i>\operatorname{dim} R / J[23$, Corollary 4.4].

(d) If $M$ is an $(I, J)$-torsion $R$-module, then $\mathfrak{F}_{\mathfrak{a}, I, J}^{i}(M)=0$ for any $i$ integer [23, Corollary 1.13].

5. The Mayer-Vietoris sequence. It is well known to readers with a basic grounding in homological algebra that an important rule is called the Mayer-Vietoris sequence. In our context, it is not known whether the Mayer-Vietoris sequence involves the local cohomology defined by a pair of ideals. The following theorem is a generalization of [19, Theorem 5.1], where Schenzel showed a similar result of the Mayer-Vietoris sequence for formal local cohomology.

Theorem 5.1. Let $\mathfrak{a}, \mathfrak{b}$ be two ideals of a local ring $(R, \mathfrak{m})$. Let $M$ be a finitely generated $R$-module. Then, for $i \in \mathbb{Z}$, there is the long exact sequence

$$
\begin{array}{r}
\cdots \longrightarrow \check{\mathfrak{F}}_{\mathfrak{a} \cap \mathfrak{b}, I, J}^{i}(M) \longrightarrow \check{\mathfrak{F}}_{\mathfrak{a}, I, J}^{i}(M) \oplus \check{\mathfrak{F}}_{\mathfrak{b}, I, J}^{i}(M) \longrightarrow \check{\mathfrak{F}}_{(\mathfrak{a}, \mathfrak{b}), I, J}^{i}(M) \\
\longrightarrow \check{\mathfrak{F}}_{\mathfrak{a} \cap \mathfrak{b}, I, J}^{i+1}(M) \longrightarrow \cdots
\end{array}
$$

Proof. Firstly, for $n \in \mathbb{Z}$, consider the following exact sequence:

$$
0 \longrightarrow M /\left(\mathfrak{a}^{n} M \cap \mathfrak{b}^{n} M\right) \longrightarrow M / \mathfrak{a}^{n} M \oplus M / \mathfrak{b}^{n} M \longrightarrow M /\left(\mathfrak{a}^{n}, \mathfrak{b}^{n}\right) M \longrightarrow 0 .
$$


It induces a short exact sequence

$$
\begin{array}{r}
0 \longrightarrow \check{C}_{\underline{x}, J} \otimes \frac{M}{\mathfrak{a}^{n} M \cap \mathfrak{b}^{n} M} \longrightarrow\left(\check{C}_{\underline{x}, J} \otimes \frac{M}{\mathfrak{a}^{n} M}\right) \oplus\left(\check{C}_{\underline{x}, J} \otimes \frac{M}{\mathfrak{b}^{n} M}\right) \\
\longrightarrow \check{C}_{\underline{x}, J} \otimes \frac{M}{\left(\mathfrak{a}^{n}, \mathfrak{b}^{n}\right) M} \longrightarrow 0 .
\end{array}
$$

Because $\check{C}_{\underline{x}, J}$ is a complex of flat $R$-modules and the maps,

$$
M /\left(\mathfrak{a}^{n+1} \cap \mathfrak{b}^{n+1}\right) M \longrightarrow M /\left(\mathfrak{a}^{n} \cap \mathfrak{b}^{n}\right) M,
$$

are surjective, we have that the projective system of $R$-complexes $\left\{\check{C}_{\underline{x}, J} \otimes M / \mathfrak{a}^{n} M \cap \mathfrak{b}^{n} M\right\}$ satisfies the Mittag-Leffler condition. Therefore, by [11, Proposition 13.2.2], we obtain the exact sequence of complexes:

$$
\begin{aligned}
0 \longrightarrow & \lim _{\longleftarrow}\left(\check{C}_{\underline{x}, J} \otimes \frac{M}{\mathfrak{a}^{n} M \cap \mathfrak{b}^{n} M}\right) \longrightarrow \lim _{\longleftarrow}\left(\check{C}_{\underline{x}, J} \otimes \frac{M}{\mathfrak{a}^{n} M}\right) \\
& \oplus \underset{\leftarrow}{\lim }\left(\check{C}_{\underline{x}, J} \otimes \frac{M}{\mathfrak{b}^{n} M}\right) \longrightarrow \lim _{\longleftarrow}\left(\check{C}_{\underline{x}, J} \otimes \frac{M}{\left(\mathfrak{a}^{n}, \mathfrak{b}^{n}\right) M}\right) \longrightarrow 0 .
\end{aligned}
$$

We observe that the $\left(\mathfrak{a}^{n}, \mathfrak{b}^{n}\right)$-adic filtration is equivalent to the filtration $\left\{\left(\mathfrak{a}^{n}, \mathfrak{b}^{n}\right) M\right\}_{n \in \mathbb{N}}$. Now it is enough to prove the (ân $\left.\mathfrak{b}\right)$-adic filtration on $M$ is equivalent to the filtration $\left\{\left(\mathfrak{a}^{n} \cap \mathfrak{b}^{n}\right) M\right\}_{n \in \mathbb{N}}$. Note that

$$
(\mathfrak{a b})^{n} M \subseteq\left(\mathfrak{a}^{n} \cap \mathfrak{b}^{n}\right) M \subseteq \mathfrak{a}^{n} M \cap \mathfrak{b}^{n} M
$$

for all $n \in \mathbb{N}$. Let $m \in \mathbb{N}$ be a given integer. By the Artin-Rees lemma [3, Chapter III, Section 3, Corollary 1], there exists a $k \in \mathbb{N}$ such that

$$
\mathfrak{a}^{n} N \cap \mathfrak{b}^{m} N \subseteq \mathfrak{a}^{n-k} \mathfrak{b}^{m} N \quad \text { for all } n \geq k
$$

Note that the $\mathfrak{a} \mathfrak{b}$-adic and the $\mathfrak{a} \cap \mathfrak{b}$-adic topology on $M$ are equivalent, and this concludes the statement.

The next result is an immediate consequence of Theorem 5.1.

Corollary 5.2. Let $\mathfrak{a}$ and $\mathfrak{b}$ be ideals of a local ring $(R, \mathfrak{m})$. Let $M$ be a finitely generated $R$-module and $i \in \mathbb{Z}$. 
(i) If $M$ is a $J$-torsion $R$-module, then there is a long exact sequence

$$
\begin{array}{r}
\cdots \longrightarrow \mathfrak{F}_{\mathfrak{a} \cap \mathfrak{b}, \mathfrak{m}, J}^{i}(M) \longrightarrow \mathfrak{F}_{\mathfrak{a}, \mathfrak{m}, J}^{i}(M) \oplus \mathfrak{F}_{\mathfrak{b}, \mathfrak{m}, J}^{i}(M) \longrightarrow \mathfrak{F}_{(\mathfrak{a}, \mathfrak{b}), \mathfrak{m}, J}^{i}(M) \\
\longrightarrow \mathfrak{F}_{\mathfrak{a} \cap \mathfrak{b}, \mathfrak{m}, J}^{i+1}(M) \longrightarrow \cdots
\end{array}
$$

(ii) If $M$ is a J-torsion $R$-module and $\sqrt{I+J}=\mathfrak{m}$, then there is a long exact sequence

$$
\begin{array}{r}
\cdots \longrightarrow \mathfrak{F}_{\mathfrak{a} \cap \mathfrak{b}, I, J}^{i}(M) \longrightarrow \mathfrak{F}_{\mathfrak{a}, I, J}^{i}(M) \oplus \mathfrak{F}_{\mathfrak{b}, I, J}^{i}(M) \longrightarrow \mathfrak{F}_{(\mathfrak{a}, \mathfrak{b}), I, J}^{i}(M) \\
\longrightarrow \mathfrak{F}_{\mathfrak{a} \cap \mathfrak{b}, I, J}^{i+1}(M) \longrightarrow \cdots
\end{array}
$$

(iii) If $M$ is Artinian $R$-module, then there is a long exact sequence

$$
\begin{array}{r}
\cdots \longrightarrow \mathfrak{F}_{\mathfrak{a} \cap \mathfrak{b}, I, J}^{i}(M) \longrightarrow \mathfrak{F}_{\mathfrak{a}, I, J}^{i}(M) \oplus \mathfrak{F}_{\mathfrak{b}, I, J}^{i}(M) \longrightarrow \mathfrak{F}_{(\mathfrak{a}, \mathfrak{b}), I, J}^{i}(M) \\
\longrightarrow \mathfrak{F}_{\mathfrak{a} \cap \mathfrak{b}, I, J}^{i+1}(M) \longrightarrow \cdots
\end{array}
$$

Proof. We will prove (i), and the other cases are analogous. Because $M$ is $J$-torsion, any quotient of $M$ is $J$-torsion too. By Corollary 2.6 and Theorem 5.1, the statement follows.

6. Local duality for a pair of ideals. The purposes of this section are to show a generalization of Grothendieck duality theorem for Gorenstein rings and give another proof of the local duality theorem for Gorenstein and Cohen-Macaulay rings. For both cases, we will use local cohomology with respect to a pair of ideals.

Let $(R, \mathfrak{m}, \mathbb{K})$ be a $d$-dimensional Cohen-Macaulay local ring with a canonical module $\omega$. Then, for $0 \leq i \leq d$, it is well known that isomorphisms,

$$
H_{\mathfrak{m}}^{i}(M)=\operatorname{Ext}_{R}^{d-i}(M, \omega)^{\vee}
$$

exist where $(-)^{\vee}=\operatorname{Hom}_{R}\left(-, E_{R}(\mathbb{K})\right)$ and $H_{\mathfrak{m}}^{d}(R) \cong \omega^{\vee}$. This result is called the local duality theorem. There is a generalization of this result in [23, Theorem 5.1] for local cohomology defined by a pair of ideals.

Lemma 6.1. Let $(R, \mathfrak{m}, \mathbb{K})$ denote a local ring, $\underline{x}=x_{1}, \cdots, x_{n}$ a system of elements of $R$ such that $\mathfrak{m}=(\underline{x})$ and $J$ an ideal of $R$. If $M$ is a finitely generated $R$-module, then, for all $i \in \mathbb{Z}$, there are isomorphisms, 


$$
H_{\mathfrak{m}, J}^{i}(M) \cong \operatorname{Hom}_{R}\left(H^{-i}\left(\operatorname{Hom}_{R}\left(M, D_{\underline{x}, J}\right)\right), E\right)
$$

where $E$ is the injective hull of $\mathbb{K}:=R / \mathfrak{m}$ and $D_{\underline{x}, J}=\operatorname{Hom}_{R}\left(\check{C}_{\underline{x}, J}, E\right)$.

Proof. Proceeding analogously, using the construction made in [20, Theorem 1.7] and replacing $D_{\underline{x}}$ by $D_{\underline{x}, J}$, we obtain the result.

Now, we will show a generalization of the Grothendieck duality theorem and, after a generalization of local duality theorem, both on Gorenstein rings.

Theorem 6.2. Let $(R, \mathfrak{m}, \mathbb{K})$ be a local ring of dimension $d, J$ a perfect ideal of $R$ of grade $t$, i.e.,

$$
\operatorname{pd}_{R} R / J=\operatorname{grade}(J, R)=t .
$$

If $R$ is a Gorenstein ring, then

$$
H_{\mathfrak{m}, J}^{i}(R)= \begin{cases}0 & \text { if } i \neq d-t \\ \bigoplus_{\substack{h \in \mathfrak{p}=d-t \\ \mathfrak{p} \in W(\mathfrak{m}, J)}} E_{R}(R / \mathfrak{p}) & \text { if } i=d-t .\end{cases}
$$

Proof. Let $I^{\bullet}$ be a minimal injective resolution of $R$. Since $R$ is Gorenstein, for each $i$, there is an isomorphism

$$
I^{i}=\bigoplus_{h t \mathfrak{p}=i} E_{R}(R / \mathfrak{p})
$$

Applying the functor $\Gamma_{\mathfrak{m}, J}(-)$ and, by [23, Proposition 1.11], the complex

$$
0 \longrightarrow \bigoplus_{\substack{h t \mathfrak{p}=0 \\ \mathfrak{p} \in W(\mathfrak{m}, J)}} E_{R}(R / \mathfrak{p}) \longrightarrow \bigoplus_{\substack{h \mathfrak{t}=1 \\ \mathfrak{p} \in W(\mathfrak{m}, J)}} E_{R}(R / \mathfrak{p}) \longrightarrow \bigoplus_{\substack{h \in \mathfrak{p}=2 \\ \mathfrak{p} \in W(\mathfrak{m}, J)}} E_{R}(R / \mathfrak{p}) \longrightarrow \cdots
$$

follows. Now, by [23, Corollary 4.4, Lemma 5.2], $H_{\mathfrak{m}, J}^{i}(R)=0$ for $i \neq d-t$ and

$$
H_{\mathfrak{m}, J}^{d-t}(R)=\bigoplus_{\substack{h t \mathfrak{p}=d-t \\ \mathfrak{p} \in W(\mathfrak{m}, J)}} E_{R}(R / \mathfrak{p})
$$

This finishes the proof. 
Theorem 6.3. Let $(R, \mathfrak{m}, \mathbb{K})$ be a Gorenstein local ring of dimension $d$, $J$ a perfect ideal of $R$ of grade $t$, i.e., $\operatorname{pd}_{R} R / J=\operatorname{grade}(J, R)=t$. If $M$ is a finitely generated $R$-module, then there are isomorphisms

$$
H_{\mathfrak{m}, J}^{i}(M) \cong \operatorname{Ext}_{R}^{d-t-i}(M, S)^{\vee}
$$

for all $0 \leq i \leq d-t$, where $(-)^{\vee}=\operatorname{Hom}_{R}\left(-, E_{R}(\mathbb{K})\right)$ and $S=$ $H_{\mathfrak{m}, J}^{d-t}(R)^{\vee}$.

Proof. Let $\underline{x}=x_{1}, \cdots, x_{n}$ be a system of elements of $R$ such that $\mathfrak{m}=(\underline{x})$. Since $H^{i}\left(\check{C}_{\underline{x}, J}\right) \cong H_{\mathfrak{m}, J}^{i}(R)$, by Theorem 6.2 , we have:

$$
H^{i}\left(\check{C}_{\underline{x}, J}\right)= \begin{cases}0 & \text { if } i \neq d-t \\ \bigoplus_{\substack{h t \mathfrak{p}=d-t \\ \mathfrak{p} \in W(\mathfrak{m}, J)}} E_{R}(R / \mathfrak{p}) & \text { if } i=d-t\end{cases}
$$

Denote

$$
\bar{E}=\bigoplus_{\substack{h t \mathfrak{p}=d-t \\ \mathfrak{p} \in W(\mathfrak{m}, J)}} E_{R}(R / \mathfrak{p})
$$

It follows that $\check{C}_{\underline{x}, J}$ is a flat resolution of $\bar{E}$ shifted $d-t$ places to the right. Therefore, $D_{\underline{x}, J}=\operatorname{Hom}_{R}\left(\check{C}_{\underline{x}, J}, E_{R}(\mathbb{K})\right)$ is an injective resolution of $\operatorname{Hom}_{R}(\bar{E}, E)$ shifted $d-t$ places to the right. Since

$$
H^{-i}\left(\operatorname{Hom}_{R}\left(M, D_{\underline{x}, J}\right)\right) \cong \operatorname{Ext}_{R}^{d-t-i}\left(M, \operatorname{Hom}_{R}(\bar{E}, E)\right)
$$

and

$$
\operatorname{Hom}_{R}(\bar{E}, E)=H_{\mathfrak{m}, J}^{i}(R)^{\vee},
$$

by Theorem 6.2, applying Lemma 6.1, we have the statement.

Note that the isomorphism in [23, Theorem 5.1] may be obtained by duality from the previous theorem.

One natural question arises: is Theorem 6.3 true when $R$ is Cohen Macaulay?

In order to answer this question, we need preliminary observations.

Let $R$ be a commutative Noetherian ring, $I$ and $J$ two ideals of $R$ and $M$ an $R$-module. Let

$$
\operatorname{depth}(I, J, M)=\inf \left\{i \in \mathbb{N}_{0} \mid H_{I, J}^{i}(M) \neq 0\right\} .
$$


If we consider $M$ to be a finitely generated module over a local ring $(R, \mathfrak{m})$ and $J \neq R$, by [23, Theorem 4.5] and the above definition, we have $H_{\mathfrak{m}, J}^{i}(M) \neq 0$ for all

$$
\operatorname{depth}(\mathfrak{m}, J, M) \leq i \leq \operatorname{dim} M / J M .
$$

This motivates the following definition.

Definition 6.4. When $\operatorname{depth}(\mathfrak{m}, J, M)=\operatorname{dim} M / J M$, the $R$-module $M \neq 0$ is called ( $\mathfrak{m}, J$ )-Cohen Macaulay (or if $M=0$ ). If $R$ itself is an $(\mathfrak{m}, J)$-Cohen-Macaulay $R$-module, we say that $R$ is an $(\mathfrak{m}, J)$-Cohen Macaulay ring.

In this definition, it is obvious that $J \neq 0$. Note that, if $J=0$, this natural definition of $(\mathfrak{m}, J)$-Cohen-Macaulay coincides with the definition of Cohen-Macaulay $R$-modules. The same definition applies for any $I, J$ two ideals of $R$, and, for more details, we recommend [1]. With these comments, we show below a generalization of the local duality theorem for Cohen-Macaulay rings, which extends Theorem 6.3.

Theorem 6.5. Let $M \neq 0$ be a finitely generated module over a local ring, $(R, \mathfrak{m}, \mathbb{K})$. Suppose that $R$ is $(I, J)$-Cohen-Macaulay, where $I+J$ is an $\mathfrak{m}$-primary ideal. Then there are isomorphisms

$$
H_{I, J}^{i}(M)^{\vee} \cong \operatorname{Ext}_{R}^{\hat{d}-i}(M, S),
$$

for all $0 \leq i \leq \widehat{d}$, where $(-)^{\vee}=\operatorname{Hom}_{R}\left(-, E_{R}(\mathbb{K})\right), S=H_{I, J}^{\hat{d}}(R)^{\vee}$ and $\widehat{d}:=\operatorname{dim}(M / J M)$.

Proof. Firstly note that, since $I+J$ is an m-primary ideal, we have $H_{I, J}^{i}(R)=H_{\mathfrak{m}, J}^{i}(R)$ for any $i$ integer [23, Proposition $\left.1.4(6),(7)\right]$, i.e., in this case, $R$ is $(I, J)$-Cohen Macaulay if and only if $R$ is $(\mathfrak{m}, J)$ Cohen-Macaulay. Thus, we may assume that $I=\mathfrak{m}$.

Let $\underline{x}=x_{1}, \ldots, x_{n}$ be a system of elements of $R$ such that $\mathfrak{m}=(\underline{x})$. Since $H^{i}\left(\check{C}_{\underline{x}, J}\right) \cong H_{\mathfrak{m}, J}^{i}(R)$, and $R$ is $(\mathfrak{m}, J)$-Cohen-Macaulay, $\check{C}_{\underline{x}, J}$ is a flat resolution of $H_{\mathfrak{m}, J}^{\hat{d}}(R)$ shifted $\widehat{d}$ places to the right because $H_{\mathfrak{m}, J}^{i}(R)=0$ for all $i \neq \widehat{d}([\mathbf{1}$, Corollary 4.13] or [23, Theorem 4.5]). 
Now, we have that

$$
\begin{aligned}
H_{\mathfrak{m}, J}^{i}(M) & \cong H^{i}\left(\check{C}_{\underline{x}, J}[-\widehat{d}] \otimes_{R} M\right) \cong H_{\hat{d}-i}\left(\check{C}_{\underline{x}, J} \otimes_{R} M\right) \\
& \cong \operatorname{Tor}_{\hat{d}-i}^{R}\left(H_{\mathfrak{m}, J}^{\hat{d}}(R), M\right)
\end{aligned}
$$

Let $K^{\bullet}$ be a free resolution of $M$. Thus, as

$$
H_{\hat{d}-i}\left(K^{\bullet} \otimes_{R} H_{\mathfrak{m}, J}^{\hat{d}}(R)\right) \cong \operatorname{Tor}_{\hat{d}-i}^{R}\left(M, H_{\mathfrak{m}, J}^{\hat{d}}(R)\right),
$$

it follows that

$$
H_{\mathfrak{m}, J}^{i}(M) \cong H_{\hat{d}-i}\left(K^{\bullet} \otimes_{R} H_{\mathfrak{m}, J}^{\hat{d}}(R)\right) .
$$

Therefore, for all integers $i$, we have

$$
\begin{aligned}
H_{\mathfrak{m}, J}^{i}(M)^{\vee} & \cong H_{\hat{d}-i}\left(K^{\bullet} \otimes_{R} H_{\mathfrak{m}, J}^{\hat{d}}(R)\right)^{\vee} \\
& \cong H^{\hat{d}-i}\left(\left(K^{\bullet} \otimes_{R} H_{\mathfrak{m}, J}^{\hat{d}}(R)\right)^{\vee}\right) \\
& \cong H^{\hat{d}-i}\left(\operatorname{Hom}_{R}\left(K^{\bullet} \otimes_{R} H_{\mathfrak{m}, J}^{\hat{d}}(R), E_{R}(\mathbb{K})\right)\right) \\
& \cong H^{\hat{d}-i}\left(\operatorname{Hom}_{R}\left(K^{\bullet}, H_{\mathfrak{m}, J}^{\hat{d}}(R)^{\vee}\right)\right) \\
& \cong \operatorname{Ext}_{R}^{\hat{d}-i}\left(M, H_{\mathfrak{m}, J}^{\hat{d}}(R)^{\vee}\right)
\end{aligned}
$$

In the following, we are interested in the characterization of formal local cohomology defined by a pair of ideals using local cohomology and the Matlis duality functor. The next result shows this relation and generalizes [19, Theorem 3.5].

Theorem 6.6. Let $(R, \mathfrak{m})$ denote a local ring, $\underline{x}=x_{1}, \ldots, x_{n}$ a system of elements of $R$ such that $\mathfrak{m}=(\underline{x})$ and $J$ an ideal of $R$. If $M$ is a finitely generated $R$-module, then, for all $i \in \mathbb{Z}$, there are the isomorphisms:

$$
\mathfrak{F}_{\mathfrak{a}, \mathfrak{m}, J}^{i}(M) \cong \operatorname{Hom}_{R}\left(H_{\mathfrak{a}}^{-i}\left(\operatorname{Hom}_{R}\left(M, D_{\underline{x}, J}\right)\right), E_{R}(\mathbb{K})\right) .
$$

Proof. By Lemma 6.1, there are isomorphisms,

$$
H_{\mathfrak{m}, J}^{i}\left(M / \mathfrak{a}^{n} M\right) \cong \operatorname{Hom}_{R}\left(H^{-i}\left(\operatorname{Hom}_{R}\left(M, D_{\underline{x}, J}\right)\right), E_{R}(\mathbb{K})\right),
$$


for all $i \in \mathbb{Z}$ and for $n \in \mathbb{N}$. Apply the projective limit and the fact that

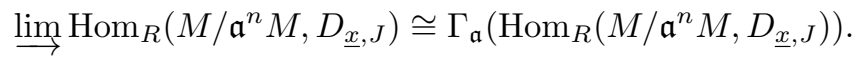

We then obtain the statement.

Remark 6.7. On the other hand, with the same hypothesis as Theorem 6.5 , we obtain

$$
\mathfrak{F}_{\mathfrak{a}, I, J}^{i}(M) \cong \operatorname{Hom}_{R}\left(\lim _{\longrightarrow} \operatorname{Ext}_{R}^{\hat{d}-i}\left(M / \mathfrak{a}^{n} M, S\right), E_{R}(\mathbb{K})\right) .
$$

Note that, for all $i \in \mathbb{Z}$,

$$
\underset{\longrightarrow}{\lim } \operatorname{Ext}_{R}^{\hat{d}-i}\left(M / \mathfrak{a}^{n} M, S\right)
$$

is exactly the generalized local cohomology with respect to $\mathfrak{a}$ (denoted by $\left.H_{\mathfrak{a}}^{\hat{d}-i}(M, S)\right)$, introduced by Herzog [12]. Therefore,

$$
\mathfrak{F}_{\mathfrak{a}, I, J}^{i}(M) \cong H_{\mathfrak{a}}^{\hat{d}-i}(M, S)^{\vee},
$$

where

$$
(-)^{\vee}=\operatorname{Hom}_{R}\left(-, E_{R}(\mathbb{K})\right), \quad i \in \mathbb{Z} .
$$

This shows the relation between the formal local cohomology defined by a pair of ideals and the Matlis dual of certain generalized local cohomology with respect to $\mathfrak{a}$.

For the next result, we firstly need the following considerations. Let $(\widehat{R}, \widehat{\mathfrak{m}})$ be the $\mathfrak{m}$-adic completion of $(R, \mathfrak{m}, \mathbb{K})$, and consider the natural homomorphism $R \rightarrow \widehat{R}$. By Theorem 2.3, we may assume the existence of the complex

$$
D_{\underline{x}, J}=\operatorname{Hom}_{R}\left(\check{C}_{\underline{x}, J}, E_{R}(\mathbb{K})\right) .
$$

For $x \in \mathfrak{m}$, we are interested in determining how the $\mathfrak{a}$-formal local cohomology and $(\mathfrak{a}, x)$-formal local cohomology, both defined by a pair of ideals, are connected. The next long exact sequence shows this relation and generalizes [19, Theorem 3.15].

Theorem 6.8. Let $(R, \mathfrak{m}, \mathbb{K})$ be a local ring, and let $\underline{x}=x_{1}, \ldots, x_{n}$ and $\underline{y}=y_{1}, \ldots, y_{n}$ be two systems of elements of $R$ such that $\mathfrak{m}=(\underline{x})$, 
$\mathfrak{a}=(y)$. If $M$ is a finitely generated $R$-module and $x \in \mathfrak{m}$ is an element of $R$, then there is the long exact sequence

$\cdots \longrightarrow \operatorname{Hom}_{R}\left(R_{x}, \mathfrak{F}_{\mathfrak{a}, \mathfrak{m}, J}^{i}(M)\right) \longrightarrow \mathfrak{F}_{\mathfrak{a}, \mathfrak{m}, J}^{i}(M) \longrightarrow \mathfrak{F}_{(\mathfrak{a}, x), \mathfrak{m}, J}^{i}(M) \longrightarrow \cdots$, for all $i \in \mathbb{Z}$.

Proof. By the previous comment, we can consider the complex $D_{\underline{x}, J}$. Let $\check{C}_{x}$ be the $\check{C}$ ech complex for an element $x \in \mathfrak{m}$. So, there is the short exact sequence of flat $R$-modules,

$$
0 \longrightarrow R_{x}[-1] \longrightarrow \check{C}_{x} \longrightarrow R \longrightarrow 0 .
$$

Denote $\widetilde{\mathrm{H}}=\operatorname{Hom}_{R}\left(M, D_{x, J}\right)$. Tensoring the previous exact sequence with $\check{C}_{\underline{y}} \otimes \widetilde{\mathrm{H}}$, we have the following exact sequence of $R$-modules

$$
0 \longrightarrow \check{C}_{\underline{y}} \otimes \widetilde{\mathrm{H}} \otimes R_{x}[-1] \longrightarrow \check{C}_{\underline{y}, x} \otimes \widetilde{\mathrm{H}} \longrightarrow \check{C}_{\underline{y}} \otimes \widetilde{\mathrm{H}} \longrightarrow 0 .
$$

Now, seeing the long exact cohomology sequence together with $[\mathbf{2 3}$, Theorem 2.4] we obtain, for all $i \in \mathbb{Z}$,

$$
\cdots \longrightarrow H_{(\mathfrak{a}, x R)}^{i}(\widetilde{\mathrm{H}}) \longrightarrow H_{\mathfrak{a}}^{i}(\widetilde{\mathrm{H}}) \longrightarrow H_{\mathfrak{a}}^{i}(\widetilde{\mathrm{H}}) \otimes R_{x} \longrightarrow \cdots .
$$

Applying the functor $\operatorname{Hom}_{R}\left(-, E_{R}(\mathbb{K})\right)$ and Theorem 6.6 we obtain the result.

The natural consequence and application of the preceding theorem is when $\mathfrak{a}=0$. The next result relates the formal local cohomology with respect to an ideal generated by a single element and local cohomology, both defined by a pair of ideals.

Corollary 6.9. With the same hypothesis as the previous theorem, there is a short exact sequence,

$\cdots \longrightarrow \operatorname{Hom}_{R}\left(R_{x}, H_{\mathfrak{m}, J}^{i}(M)\right) \longrightarrow H_{\mathfrak{m}, J}^{i}(M) \longrightarrow \mathfrak{F}_{x R, \mathfrak{m}, J}^{i}(M) \longrightarrow \cdots$, for all $i \in \mathbb{Z}$.

7. Formal grade with respect to a pair of ideals. The concept of formal grade was introduced by Peskine and Szpiro [18], and not much is known about this tool. In our approach, since

$$
\mathfrak{F}_{\mathfrak{a}, I, J}^{i}(M) \cong \check{\mathfrak{F}}_{\mathfrak{a}, I, J}^{i}(M)
$$


for some cases, we need to give two definitions for formal grade, different from the approach given by Schenzel [19].

Definition 7.1. For an ideal $\mathfrak{a}$ of $R$ we define

$$
\text { fgrade }(\mathfrak{a}, I, J, M)=\inf \left\{i \in \mathbb{Z} \mid \mathfrak{F}_{\mathfrak{a}, I, J}^{i}(M) \neq 0\right\}
$$

and

$$
\check{f} \text { grade }(\mathfrak{a}, I, J, M)=\inf \left\{i \in \mathbb{Z} \mid \check{\mathfrak{F}}_{\mathfrak{a}, I, J}^{i}(M) \neq 0\right\} .
$$

Note that

$$
\text { fgrade }(\mathfrak{a}, I, J, M) \geq \check{f} \text { grade }(\mathfrak{a}, I, J, M),
$$

by Proposition 2.4. The next two results extend [15, Proposition 2.2, Corollary 2.4].

Proposition 7.2. Let $(R, \mathfrak{m})$ be a local ring. If

$$
0 \longrightarrow P \longrightarrow M \longrightarrow N \longrightarrow 0
$$

is an exact sequence of finitely generated $R$-modules, then the following statements hold.

$$
\check{f} \text { grade }(\mathfrak{a}, I, J, M) \geq \min \{\check{f} \text { grade }(\mathfrak{a}, I, J, P), \check{f} \text { grade }(\mathfrak{a}, I, J, N)\} \text {. }
$$

$\check{f}$ grade $(\mathfrak{a}, I, J, P) \geq \min \{\check{f}$ grade $(\mathfrak{a}, I, J, M), \check{f} \operatorname{grade}(\mathfrak{a}, I, J, N)+1\}$.

$\check{f}$ grade $(\mathfrak{a}, I, J, N) \geq \min \{\check{f}$ grade $(\mathfrak{a}, I, J, P)-1, \check{f}$ grade $(\mathfrak{a}, I, J, M)\}$.

(iv) One of the following equalities holds:

$$
\begin{gathered}
\check{f} \text { grade }(\mathfrak{a}, I, J, M)=\check{f} \text { grade }(\mathfrak{a}, I, J, P), \\
\check{f} \text { grade }(\mathfrak{a}, I, J, M)=\check{f} \text { grade }(\mathfrak{a}, I, J, N), \\
\check{f} \operatorname{grade}(\mathfrak{a}, I, J, M)=\check{f} \operatorname{grade}(\mathfrak{a}, I, J, N)=\check{f} \operatorname{grade}(\mathfrak{a}, I, J, P), \\
\check{f} \operatorname{grade}(\mathfrak{a}, I, J, N)=\check{f} \operatorname{grade}(\mathfrak{a}, I, J, P)-1 .
\end{gathered}
$$


Proof. By Theorem 3.4, it follows that $\cdots \longrightarrow \check{\mathfrak{F}}_{\mathfrak{a}, I, J}^{i}(P) \longrightarrow \check{\mathfrak{F}}_{\mathfrak{a}, I, J}^{i}(M) \longrightarrow \check{\mathfrak{F}}_{\mathfrak{a}, I, J}^{i}(N) \longrightarrow \check{\mathfrak{F}}_{\mathfrak{a}, I, J}^{i+1}(P) \longrightarrow \cdots$ Then, we have assertions (i), (ii) and (iii). Denote

$$
\begin{gathered}
\check{f} \text { grade }(\mathfrak{a}, I, J, M)=t, \\
\check{f} \text { grade }(\mathfrak{a}, I, J, N)=s
\end{gathered}
$$

and

$$
\check{f} \text { grade }(\mathfrak{a}, I, J, P)=r .
$$

To prove (iv), suppose that none of the equalities occur. Thus, one of the following cases occurs:
(a) $r<s<t$,
(d) $t<s<r$
(b) $s<r<t$,
(e) $s<t<r$
(c) $t<r<s$,
(f) $r<t<s$.

We will show just two cases, and the others follow by the same idea. If $r<s<t$, then $s+1 \leq t$. Therefore by (ii), $s+1 \leq r<s$ and this is a contradiction.

If $s<r<t$, then $s \leq r-1<t$. Thus, by (iii), we have $r-1 \leq s$. This is a contradiction because $s=r-1$.

Corollary 7.3. Let $\mathfrak{a}, \mathfrak{b}$ be two ideals of $R$. Then:

(i) $\check{f}$ grade $(\mathfrak{a} \cap \mathfrak{b}, I, J, M) \geq \min \{\check{f}$ grade $(\mathfrak{a}, I, J, M), \check{f}$ grade $(\mathfrak{b}, I, J, M)$, $\check{f} \operatorname{grade}((\mathfrak{a}, \mathfrak{b}), I, J, M)+1\}$.

(ii) $\check{f}$ grade $((\mathfrak{a}, \mathfrak{b}), I, J, M) \geq \min \{\check{f}$ grade $(\mathfrak{a} \cap \mathfrak{b}, I, J, M)-1, \check{f}$ grade $(\mathfrak{a}, I$, $J, M), \check{f}$ grade $(\mathfrak{b}, I, J, M)\}$.

Proof. By Theorem 5.1, there is the long exact sequence

$$
\begin{array}{r}
\cdots \longrightarrow \check{\mathfrak{F}}_{\mathfrak{a} \cap \mathfrak{b}, I, J}^{i}(M) \longrightarrow \check{\mathfrak{F}}_{\mathfrak{a}, I, J}^{i}(M) \oplus \check{\mathfrak{F}}_{\mathfrak{b}, I, J}^{i}(M) \longrightarrow \check{\mathfrak{F}}_{(\mathfrak{a}, \mathfrak{b}), I, J}^{i}(M) \\
\longrightarrow \check{\mathfrak{F}}_{\mathfrak{a} \cap \mathfrak{b}, I, J}^{i+1}(M) \longrightarrow \cdots
\end{array}
$$

Therefore, analogously to Proposition 7.2, the result follows. 
Remark 7.4. It is clear that $\check{f}$ grade $(\mathfrak{a}, I, J, M)=$ fgrade $(\mathfrak{a}, I, J, M)$, for all cases in Corollary 2.6.

The reader can compare the next result with [2, Corollary 4.2] and [19, Lemma 4.8 (d)].

Theorem 7.5. Let $(R, \mathfrak{m}, \mathbb{K})$ be a Cohen-Macaulay complete local ring of dimension $d$, and let $J \neq 0$ be a perfect ideal of $R$ of grade $t$, i.e., $\operatorname{pd}_{R}(R / J)=\operatorname{grade}(J, R)=t$. Then, for $M$ a finitely generated $R$ module,

$$
\text { fgrade }(\mathfrak{a}, \mathfrak{m}, J, M)+\operatorname{cd}_{\mathfrak{a}}(M, S)+\operatorname{grade}(J, R)=\operatorname{dim} R,
$$

where $S=H_{\mathfrak{m}, J}^{d-t}(R)^{\vee}$.

Proof. By Theorem 6.5, we have that

$$
H_{\mathfrak{m}, J}^{i}(M) \cong \operatorname{Hom}_{R}\left(\operatorname{Ext}_{R}^{d-t-i}(M, S), E_{R}\right) .
$$

So,

$$
\begin{aligned}
\mathfrak{F}_{\mathfrak{a}, m, J}^{i}(M) & =\lim _{\mathfrak{m}, J} H_{\mathfrak{m}, J}^{i}\left(M / \mathfrak{a}^{n} M\right) \\
& \cong \lim _{R} \operatorname{Hom}_{R}\left(\operatorname{Ext}_{R}^{d-t-i}\left(M / \mathfrak{a}^{n} M, S\right), E_{R}(\mathbb{K})\right) \\
& =\operatorname{Hom}_{R}\left(\underline{\lim }_{\longrightarrow} \operatorname{Ext}_{R}^{d-t-i}\left(M / \mathfrak{a}^{n} M, S\right), E_{R}(\mathbb{K})\right) .
\end{aligned}
$$

Since

$$
H_{\mathfrak{a}}^{i}(M, S)=\lim _{\longrightarrow} \operatorname{Ext}_{R}^{d-t-i}\left(M / \mathfrak{a}^{n} M, S\right)
$$

[12], for all $i \in \mathbb{Z}$, there are isomorphisms

$$
\mathfrak{F}_{\mathfrak{a}, m, J}^{i}(M) \cong \operatorname{Hom}_{R}\left(H_{\mathfrak{a}}^{d-t-i}(M, S), E_{R}(\mathbb{K})\right)
$$

Therefore,

$$
\begin{aligned}
\inf \left\{i \in \mathbb{Z} \mid \mathfrak{F}_{\mathfrak{a}, \mathfrak{m}, J}^{i}(M) \neq 0\right\} & =\inf \left\{i \in \mathbb{Z} \mid H_{\mathfrak{a}}^{d-t-i}(M, S) \neq 0\right\} \\
& =\inf \left\{d-t-j \mid H_{\mathfrak{a}}^{j}(M, S) \neq 0\right\} \\
& =d-t-\sup \left\{j \mid H_{\mathfrak{a}}^{j}(M, S) \neq 0\right\} \\
& =\operatorname{dim} R-\operatorname{grade}(J, R)-\operatorname{cd}_{\mathfrak{a}}(M, S) .
\end{aligned}
$$

An example that makes use some of results obtained in this work follows. The reader can compare this example with [19, Lemma 5.4]. 
Example 7.6. Let $(R, \mathfrak{m})$ be a complete local Noetherian ring, $\underline{x}=$ $x_{1}, \ldots, x_{s}$ a system of elements of $R, I=(\underline{x}), J$ two ideals of $R$ and $M$ a finitely generated $R$-module.

(i) If $M$ is a $J$-torsion indecomposable $R$-module and, for an ideal a of $R$ fgrade $(\mathfrak{a}, \mathfrak{m}, J, M) \geq 2$, then

$$
\operatorname{Supp}_{R} M / \mathfrak{a} M \backslash\{\mathfrak{m}\}
$$

is connected.

(ii) If $M$ is a $J$-torsion indecomposable $R$-module, $\sqrt{I+J}=\mathfrak{m}$ and fgrade $(\mathfrak{a}, I, J, M) \geq 2$ for an ideal $\mathfrak{a}$ of $R$, then

$$
\operatorname{Supp}_{R} M / \mathfrak{a} M \backslash\{\mathfrak{m}\}
$$

is connected.

Proof. For the proof of (i), by contradiction, suppose the set $\operatorname{Supp}_{R} M / \mathfrak{a} M \backslash\{\mathfrak{m}\}$ is disconnected. This means that the ideals $L$ and $K$ exist in $R$, such that

(a) $L \cap K \subseteq \sqrt{(0)}$;

(b) $\sqrt{L+K}=\mathfrak{m}$;

(c) $\sqrt{L} \neq \mathfrak{m}$ and $\sqrt{K} \neq \mathfrak{m}$.

By the Mayer-Vietoris sequence (Corollary 5.2 (i)) and the hypothesis that fgrade $(\mathfrak{a}, \mathfrak{m}, J, M) \geq 2$, we have

$$
\mathfrak{F}_{L, \mathfrak{m}, J}^{0}(M) \oplus \mathfrak{F}_{K, \mathfrak{m}, J}^{0}(M) \simeq \mathfrak{F}_{(L, K), \mathfrak{m}, J}^{0}(M) .
$$

By item (b) and Proposition 4.1 (i) it follows that

$$
\mathfrak{F}_{(L, K), \mathfrak{m}, J}^{0}(M) \simeq M .
$$

Since $M$ is indecomposable, we have, without loss of generality,

$$
\mathfrak{F}_{L, \mathfrak{m}, J}^{0}(M) \simeq M \quad \text { and } \quad \mathfrak{F}_{K, \mathfrak{m}, J}^{0}(M)=0 .
$$

Furthermore, since $M$ is a $J$-torsion $R$-module, by Proposition 2.2, we have that

$$
\mathfrak{F}_{L, \mathfrak{m}, J}^{0}(M)=\mathfrak{F}_{L, \mathfrak{m}}^{0}(M)
$$


and

$$
\mathfrak{F}_{K, \mathfrak{m}, J}^{0}(M)=\mathfrak{F}_{K, \mathfrak{m}}^{0}(M) .
$$

It follows by [19, Lemma 4.1 (b), (c)] that

$$
\operatorname{Ass}_{R} M=\left\{\mathfrak{p} \in \operatorname{Ass}_{R} M: \operatorname{dim} R /(\mathfrak{p}, L)=0\right\} .
$$

Therefore,

$$
\mathfrak{m}=\bigcap_{\mathfrak{p} \in \operatorname{Ass}_{R} M} \operatorname{Rad}(L, \mathfrak{p})=\operatorname{Rad} L,
$$

but this does not happen by item (c).

Using [23, Proposition 1.4 (6), (7)] and Corollary 5.2 (ii) the proof of item (ii) is analogous.

Acknowledgments. The authors would like to thank the referee for his or her carefully reading of this manuscript and the helpful suggestions. Moreover, the authors are grateful to Professors B. Ulrich and G. Caviglia for some conversations, and for hospitality by the Department of Mathematics at Purdue University.

\section{REFERENCES}

1. M. Aghapournahr, KH. Ahmadi-Amoli and M.Y. Sadegui, The concept of $(I, J)$-Cohen-Macaulay modules, Journal of Algebraic Systems, accepted.

2. M. Asgharzadeh and K. Divaani-Aazar, Finiteness properties of formal local cohomology modules and Cohen- Macaulayness, Comm. Algebra 39 (2011), 10821103.

3. N. Bourbaki, Algébre commutative, Hermann, Paris, 1961-1965.

4. M.P. Brodmann and R.Y. Sharp, Local cohomology, An algebraic introduction with geometric applications, Cambridge University Press, Cambridge, 1998.

5. L. Chu and Q. Wang, Some results on local cohomology modules defined by a pair of ideals, J. Math. Kyoto Univ, 49 (2009), 193-200.

6. K. Divaani-Aazar, R. Naghipour and M. Tousi, Cohomological dimension of certain algebraic varieties, Proc. Amer. Math. Soc. 130 (2002), 3537-3544.

7. K. Divaani-Aazar and P. Schenzel, Ideal topology, local cohomology and connectedness, Math. Proc. Cambr. Philos. Soc. 131 (2001), 211-226.

8. M. Eghbali, On formal local cohomology, colocalization and endomorphism ring of top local cohomology modules, Ph.D. thesis, Universitat Halle-Wittenberg, 2011.

9. G. Faltings, Algebraization of some formal vector bundles, Ann. Math. 110 (1979), 501-514. 
10. A. Grothendieck, Local cohomology, Notes by R. Hartshorne, Lect. Notes Math. 20, Springer, Berlin, 1966.

11. A. Grothendieck and J. Dieudonné, Éléments de géométrie algébrique III, Publ. Math. IHES 11 (1961).

12. J. Herzog, Komplexe, Auflsungen und Dualitt in der lokalen Algebra, Habilitationsschrift, Universität Regensburg, 1970.

13. C. Huneke, Problems on local cohomology, in Free resolutions in commutative algebra and algebraic geometry, Res. Notes Math. 2 (1992), 93-108.

14. S.B. Iyengar, G.J. Leuschke, A. Leykin, C. Miller, E. Miller, A.K. Singh and U. Walther, Twenty-four hours of local cohomology, Grad. Stud. Math. 87, American Mathematical Society, 2007.

15. A. Kianezhad, A.J. Taherizadeh and A. Tehranian, Formal local cohomology modules and serre subcategories, J. Sci. Kharazmi University 13 (2013), 337-346.

16. A. Mafi, Some results on the local cohomology modules, Arch. Math (Basel) 87 (2006), 211-216.

17. _ Results on formal local cohomology modules, Bull. Malays. Math. Sci. Soc. 36 (2013), 173-177.

18. C. Peskine and L. Szpiro, Dimension projective finie et cohomologie locale, Publ. Math. I.H.E.S. 42 (1972), 47-119.

19. P. Schenzel, On formal local cohomology and connectedness, J. Alg. 315 (2007), 897-923.

20. , On the use of local cohomology in algebra and geometry, in Six lectures in commutative algebra, J. Elias, J.M. Giral, R.M. Miró-Roig and S. Zarzuela, eds., Progr. Math. 166, Birkhäuser, Berlin, 1998.

21. ( Proregular sequences, local cohomology, and completion, Math. Scand. 92 (2003), 161-180.

22. A. Tehranian and A.P.E Talemi, Non-Artinian local cohomology with respect to a pair of ideals, A Colloquium 20 (2013), 637-642.

23. T. Takahashi, Y. Yoshino and T. Yoshizawa, Local cohomology based on a nonclosed support defined by a pair of ideals, J. Pure Appl. Alg. 213 (2009), $582-600$.

24. C.A. Weibel, An introduction to homological algebra, Cambridge University Press, Cambridge, 1994.

Universidade Tecnológica Federal do Paraná-Guarapuava-PR, Brazil Email address: tfreitas@utfpr.edu.br

Universidade de São Paulo-ICMC, Caixa Postal 668, 13560-970, São CarlosSP, BRAZIL

Email address: vhjperez@icmc.usp.br 